\title{
Lapurdum
}

LAPURDUM Euskal ikerketen aldizkaria | Revue d'études basques |

Revista de estudios vascos | Basque studies review

$8 \mid 2003$

Numéro VIII

\section{Les fors basques et les droits de l'homme}

\section{Maïté Lafourcade}

\section{OpenEdition}

\section{Journals}

Édition électronique

URL : http://journals.openedition.org/lapurdum/1149

DOI : 10.4000/lapurdum. 1149

ISSN : 1965-0655

Éditeur

IKER

Édition imprimée

Date de publication : 1 novembre 2003

Pagination : 329-348

ISBN : 9782867813436

ISSN : $1273-3830$

\section{Référence électronique}

Maïté Lafourcade, «Les fors basques et les droits de l'homme », Lapurdum [En ligne], 8 | 2003, mis en ligne le 01 mai 2009, consulté le 30 avril 2019. URL : http://journals.openedition.org/lapurdum/1149 ; DOI : 10.4000/lapurdum. 1149 


\section{Maïté Lafourcade}

Professeur émérite d'Histoire du droit

Université de Pau et des Pays de l'Adour

Faculté pluridisciplinaire de Bayonne-Anglet-Biarritz

\section{Les fors basques et les droits de l'homme}

La notion de Droits de l'homme, en gestation dans l'œuvre des théologiens au Moyen Age, reprise au XVIe siècle par les juristes de l'école du droit naturel, puis développée par les philosophes au XVIIIe siècle, a suivi, en France, les progrès de l'autorité monarchiquel, pour triompher avec la Révolution française.

Si on a éprouvé le besoin en France comme en Angleterre ou en Virginie de déclarer que l'homme avait des droits fondamentaux, c'est parce qu'ils étaient violés. Ils continuèrent d'ailleurs de l'être après les déclarations, parce qu'ils avaient été conçus par une élite politique et qu'ils étaient exprimés en termes abstraits.

En Pays basque, au contraire, ils étaient innés, réellement naturels. Les Basques ne les concevaient pas comme des droits, mais comme des éléments concrets de leur culture, tenue de toute antiquité de leurs ancêtres. Ils faisaient partie de leur organisation sociale que, épris de liberté, ils s'efforcèrent, tout au long de leur histoire, de préserver.

Lorsqu'ils furent contraints d'accepter une domination étrangère, craignant son impérialisme culturel, ils rédigèrent leurs coutumes ancestrales et

I La monarchie espagnole fut plus libérale que la monarchie française, jusqu'à l'avènement des Bourbons en 1700, avec Philippe V, petit-fils de Louis XIV.

Tous les textes en faveur des droits de l'homme en Espagne, depuis le Concile de Tolède de 638, ont été publiés par Grégorio PECES-BARBA MARTINEZ, Texios básicos sobre derechos humanos, Madrid, Univ. Complutense 1973. 
exigèrent de la puissance dominatrice le serment de les respecter ${ }^{2}$.

Ce sont ces recueils de coutumes qui sont appelés Fueros ou Fors en langue française. Mais ce terme recouvre deux sens différents ${ }^{3}$. Il peut aussi désigner des privilèges octroyés par le souverain. Dans le haut Moyen-âge, il correspondait au droit appliqué par les tribunaux qui jugeaient sur la place publique. Après la renaissance du droit romain au XIIe siècle, on opposait le terme Fueros qui désignait le droit coutumier local, à celui de Derecho, droit, qui était le nouveau droit romano-canonique. Puis, avec le développement de la législation royale, les premiers actes législatifs du roi, qui étaient des chartes de privilèges concédés aux villes renaissantes, furent aussi appelés Fueros; mais ces derniers ne contenaient aucune norme de droit privé, leur contenu étant seulement de droit public. Bien qu'exprimant de nombreuses garanties personnelles, comme la plupart des chartes de peuplement, elles intéressent moins notre sujet que les recueils de coutumes qui sont d'émanation populaire.

Ces Fueros ou Coutumiers sont issus directement du peuple, sans orientation systématique, sans aucune motivation sinon la conservation des usages ancestraux, sans prétention de les rationaliser et de les traduire en formules abstraites. Si les lois forales répondent aux mêmes exigences humaines, aux mêmes inquiétudes que les déclarations des derniers siècles, elles déclarent des droits concrets.

N'ayant formé d'unité politique que pendant une courte période, sous le règne de Sanche III le Grand, roi de Navarre de 1004 à 1035, les sept ter-

\footnotetext{
2 « Lorsque la Seigneurie était autonome et souveraine, jusqu'en 1379, la Biscaye ne sentit pas la nécessité de rédiger ses usages et coutumes; mais à partir de cette date, étant intégrée dans l'union avec la Castille, les rois nommèrent des corregidores (analogues aux intendants), qui ignoraient la foralité. C'est pourquoi les Juntas en 1452 nommèrent une commission pour se rendre auprès du corregidor, Pedro Gonzalez de Santo Domingo et lui dire que, comme il le savait, la Biscaye avait des usages, coutumes, fueros et privilèges qui n'étaient pas écrits et qu'il en résultait beaucoup d'inconvénients, lui demandant qu'il les autorise à les écrire...": Adrián CELAYA IBARRA, "Los fueros vascos y los derechos humanos", Conférence Real Sociedad Bascongada de los Amigos del País, Madrid, 26 octobre 2000.
}

Cf. au sujet du pacte entre le roi et le peuple dans les différents territoires basques: Gregorio MONREAL CIA, "Algo más sobre el pactismo político", in El derecho historico vasco y su originalidad, Univ. Deusto, Forum Deusto, Quinto ciclo: Cultura vasca, Bilbao 1993.

${ }^{3}$ Cf. Adrián CELAYA IBARRA, Derecho foral y autonómico vasco, Tomo I: Derecho foral, Univ. Deusto, Bilbao 1984, p. 8-15; Antonio BERISTAIN, María Angeles LARREA et Rafael María MIEZA, Fuentes de Derecho penal vasco (siglos XI-XVI), La gran Enciclopedia vasca, Antropología vasca, vol. V, Bilbao 1980, p. 23-24. 
ritoires peuplés de Basques ${ }^{4}$ ont eu une évolution historique différente et la rédaction de leurs Fueros s'échelonne du XIIIe au XVIIe siècle ${ }^{5}$.

Le premier fut le Fueros general de Navarra qui date de 1237 6. La Navarre était une monarchie depuis le IXe siècle, les Navarrais s'étant donné un chef militaire pour repousser les Francs venus du nord et les Maures du Sud. Mais ils prirent la précaution, avant de l'élire, de lui faire jurer le respect de leurs fueros. Et, lorsqu'en 1234, un prince inconnu, venu du nord de la Loire, pays de forte féodalité, Thibaut de Champagne, hérita de la Couronne de Pampelune, les Navarrais rédigèrent leurs fueros et exigèrent du nouveau roi le serment de les respecter. Ce serment fut, par la suite, renouvelé à chaque changement de règne 7

Les trois autres territoires basques en Espagne, qui forment l'actuelle Communauté autonome basque, le Guipuzcoa, l'Alava et la Biscaye, furent détachés de la Navarre et unis au royaume de Castille, respectivement en 1200, 1332 et 1379. La province de Guipuzcoa et l'Hermandad ${ }^{8}$ d'Alava furent officiellement soumises au droit castillan. Mais les Hermandades des deux provinces promulguaient des ordonnances, essentiellement pénales, destinées à mettre fin aux luttes intestines entre bandes rivales, et le roi de Castille, reconnaissant le particularisme de ces territoires, délivrait aussi des ordonnances particulières dans lesquelles leurs usages étaient respectés et: concédés sous forme de privilèges ${ }^{9}$. La dernière codification de ces ordon-

\footnotetext{
4 Navarre, Biscaye, Alava, Guipuzcoa, Labourd, Soule et Basse-Navarre

5 Cf. Adrián CELAYA IBARRA, Derecho civil vasco, Univ. Deusto, Bilbao 1993, p. 19-31.
}

6 La date de la rédaction du Fueros general de Navarra, est controversée; certains auteurs donnent la date de 1238. De toute façon, elle eut lieu pendant le règne de Thibaut de Champagne, au XIIJe siè-. cle. Il fut plusieurs fois remanié, notamment en 1330 et en 1418. La Novíssima Recopilación date de 1735, et la demière actualisation du $1^{\text {er }}$ mars 1973, modifiée par la loi du ler avril 1987. Cf. sur le droit foral navarrais: Francisco SALINAS QUIJADA, Estudios de Historia del Derecho foral de Navarra, Deputación foral de Navarra, Pamplona 1977; Juan F. UTRILLA UTRILLA, El fuero general de Navarra, Pamplona 1987 (2 vol.).

7 " Carta jurada del rey Teobaldo II reconociendo las libertades de Navarra. I noviembre 1253". Gregorio PECES-BARBA MARTINEZ, op.-cit, p. 28-30.

8 Hermandad vient de hermano: frère et peut se traduire par fraternité, confrérie se traduisant par Cofradía. Ces associations pour lutter contre les bandes armées et rétablir la paix furent nombreuses au Moyen-âge dans toutes les provinces basques aussi bien qu'en Aragon et en Castille. Cf. pour le Labourd, Maité LAFOURCADE, "Une confrérie originale au Moyen-âge: l'Armandat du Pays. de Labourd", Lapurdum II, p.293-301.

9 « Privilegio de contrato", concédé à l'Alava, paz Alphonse XI en 1332, les Alavais ayant de leur plein gré accepté l'union avec la Castille, mais à la condition de conserver leurs fueros. En 1463, furent rédigés, au sein d'une junta (assemblée générale), les 60 articles qui constituent la base du droit public alavais et qui furent complétés par des ordonnances royales, compilées en 1761, puis en 1825, et par les décisions des juntas de la province. Cf. Amalio MARICHALAR et Cayetano MANRIQUE, Historia de la legislación y recitaciones del derecho civil de España. Fueros deNavarra, Vizcaya, Guipúzcoa y Alava, Madrid 1868, San Sebastián 1971. 
nances, en Guipuzcoa, date de 1696 et porte le nom de Fuero de Guipuzcoa 10. Bien que ces ordonnances ne concernent que le droit public et le droit pénal, elles reconnaissent à ces deux provinces les mêmes franchises et libertés qu'en Biscaye.

En Alava, seule la. vallée d'Ayala, au nord-ouest de la provincé, fit rédiger ses antiques usages; le Fuero de Ayala date de 1373 11. En Biscaye, les Juntas generales, assemblée représentative du peuple biscayen 12 rédigèrent: le Fuero Viejo en 1452, puis le Fuero Nuevo en 152613.

Comme le roi de Navarre, le roi de Castille, seigneur de Biscaye, prêtait serment, à chaque changement de règne, sous l'arbre de Guernika, symbole de la liberté du peuple biscayen, de respecter ses fueros ${ }^{14}$.

Le serment du roi était sanctionné par une institution appelée derecho de sobrecarta en Navarre, uso en Guipuzcoa et pasé foral en Biscaye. C' était le droit pour le peuple représenté par les juntas de vérifier si les ordonnances royales ne portaient pas atteinte aux fuero; si elles les estimaient contra fueros, elles adressaient des observations au roi, et si celui-ci ne voulait pas modifier son texte de loi, il était enregistré avec cette formule remarquable: "Se obedece, pero no se cumple" (on obéit, mais on n'exécute pas) 15. Ce droit était analogue au droit d'enregistrement et de remontrances des parlements en France, mais avec cette notable différence: en Pays basque, le dernier mot appartenait au peuple.

10 Les "Cuadernos de Hermandad de Guipúzcoa", première rédaction de coutumes, date de 1375. Elles furent suivies de nombreuses autres rédactions, notamment en 1397 (ordonnances de Guétaria), 1453, 1463, 1467, 1469, 1470, 1482..., 1527. La Nueva recopilación des Fueros de Guipuzcoa date de 1696. Cf: : Antonio CILLAN APALATEGUI, la Foralidad guipuzcoana, Caja de Ahorros provincial de Guipuzcoa, 1969.

II La seigneurie d’Ayala n'adhéra à la Hermandad alavaise qu'en 1463; alors que ses fueros avaient déjà été rédigés. Cf. Jésús GALINDEZ SUAREZ, La M.N.Y.M.L. Tierra de Ayala, su señorio y su fuero, Madrid 1933.

12 Les Juntas étaient composées des représentants des paroisses, conseils et villes. Ces entités locales réunissaient tous les habitants dans une assemblée pour élire les représentants aux Juntas genérales de Biscaye, qui se tenaient à Guernica. Cf. au sujet des Juntas de Biscaye: Gregorio MONREAL CIA, Las insituciones públicas del Señorio de Viscaya, Deputación de Viscaya, Bilbao 1974 , p. 329-409.

\section{Legislación foral de Bizkaia, Diputación foral de Bizkaia, Bilbao 1991.}

14 Chapitre I du Fuero de 1452: "Comment et de quelle manière le seigneur de Biscaye doit jurer", et loi I du titre I du Fueros Nuevo de 1526: "Comment le seigneur de Biscaye, quand il hérite, ou succède dans la Seigneurie, doit venir prêter serment". Ce serment subsista jusqu'à la fin de la première guerre carliste en 1840 .

15 Loi XI du titre I du Fuero Nuevo. 
Quant aux provinces basques françaises, le Labourd et la Soule après avoir été dans la mouvance du roi d'Angleterre depuis le mariage en 1152 d'Aliénor d'Aquitaine avec Henri Plantagenet, devenu roi deux ans plus tard à la mort de son père furent conquises par Charles VII en 1450 et intégrées au royaume de France. Leurs coutumes furent rédigées après l'ordonnance de Montils-les-Tours de 1454 , en 1514 pour le Labourd et 1520 pour la Soule 16.

La Basse-Navarre ou merindad de ultra puertos, district du royaume de Navarre, ne faisait pas partie du royaume de Navarre lorsque Charles VII promulgua l'ordonnance de Montils-les-Tours. Seule possession que Charles Quint laissa en 1530 à Henri II d'Albret, elle continua à être régie par le Fueros general de Navarra et par les usages locaux, jusqu'à ce qu'Henri IV, roi de France et de Navarre, ordonnât en 1608 la rédaction des coutumes de ce petit royaume. Le For de Basse-Navarre fut achevé en 1611 ; mais la rédaction ayant été réalisée par une commission de juristes nommés par le roi et le serment du roi n'y figurant pas, les Etats de Basse-Navarre adressèrent des remontrances au roi 17 . Leurs revendications furent vaines et les "Fors et Coutumes du Royaume de Navarre deça-ports" furent enregistrées par la Chancellerie de Navarre en 1622, deux ans après l'annexion d'autorité du royaume de Navarre à celui de France par Louis XIII en $1620^{18}$. Il ne fut édité qu'en 1645.

La monarchie française avait définitivement intégré les trois provinces basques dans le royaume de France et la justice royale s'y était imposée. On décèle cependant dans les trois recueils de coutumes, des manifestations de ce que le langage moderne dénomme "droits de l'homme". Mais c'est surtout dans les Fueros espagnols, notamment dans ceux de Biscaye, le Fuero viejo de 1452 et le Fueros nuevo de 1526, que sont le plus nettement exprimés le respect des libertés individuelles et l'égalité juridique.

\section{Les libertés individuelles}

Les provinces basques, à l'exception du royaume de Navarre et de l'Alava, terre de reconquête, n'ont guère connu la féodalité, sinon très tardi-

16 Les coutumes de Soule, Labourd et Basse-Navarre ont été publiées par Pierre HARISTOY, Recherches historiques sur le Pays basque, Bayonne 1883-1884 (2 volumes), Tome II, p. 381 à 561.

${ }^{17} \mathrm{Cf}$. au sujet de la procédure de rédaction et des remontrances des Etats de Navarre, Jean GOYHENETCHE, For et coutumes de Basse Navarre, Elkar, Bayonne 1985, p. 39-58.

18 Cf. Alain DESTRÉE, La Basse-Navarre et ses institutions de 1620 à la Révolution, thèse Droit Paris 1954, p. 40-43. 
vement et imparfaitement. Les hommes étaient de condition libre, et en Espagne, jouissaient d'étonnantes garanties judiciaires.

\section{A. La liberté personnelle}

Propriétaires des terres qu'ils cultivaient, les Basques étaient et demeurèrent de condition libre. Le servage était inconnu, sinon en Navarre et en Alava.

Cette liberté personnelle est affirmée dans les Fueros et dans les Coutumiers. Les Coutumes des trois provinces basques françarses lui consacrent un chapitre spécial, le premier dans la coutume de Soule qui expose dès son premier article "Par la Coutume observée et gardée de toute antiquité, tous les natifs et habitants de la terre sont libres et de franche condition, sans tâche de servitude...". Suivent, en cinq articles, les privilèges découlant de cette condition : exemption de service militaire et de droits sur leur personne ${ }^{19}$, liberté matrimoniale et testamentaire, libre circulation ${ }^{20}$, droit de port d'armes ${ }^{21}$, droit de s'assembler librement pour traiter de leurs affaires communes et de faire des règlements 22 qui s'imposent à tous 23 . "Et si quelqu'un attente à leurs droits, le Roi doit les défendre et les garder en leur franchise" 24

Ces mêmes droits sont reconnus aux Labourdins dans le dernier chapitre de leur Coutume, consacré aux "Franchises et libertés du pays et habitants du Labourd". Le For de Basse-Navarre, dans sa demière rubrique inti-

\footnotetext{
19 «... Personne ne peut lever aucune troupe de gens demeurant dans ladite terre. Personne non plus ne peut exiger aucun droit du fait de la personne et du corps des dits manants et habitants et d'aucun d'eux.": art I du titre I de la Coutume de Soule (traduction du texte gascon de l'édition de 1760 par Michel GROSCLAUDE, La Coutume de Soule, Baigorry 1993.

20 « Les natifs et habitants de ladite terre de Soule peuvent se marier, prendre des dispositions testamentaires et être ordonnés clercs et sortir duditpays... » : art. II du titre I de la Coutume deSoule.

21 « Les habitants de Soule, du fait qu'ils sont situés à l'extrémité du Royaume, entourés et enfermés par les Royaumes de Navarre et d'Aragon et le pays de Béarn, ont le droit de porter leurs armes en tout temps pour leur défense et celle dudit pays...": art. III du titre I de la Coutume de Soule.

22 "Les paroissiens de chaque paroisse dudit pays, et degairies de Soule peuvent s'assembler pour traiter de leurs besognes communes et celles de leurs paroisses, vics et dégairies, chaque fois que besoin est. Ils peuvent faire el ordonner entre eux des statuts et ordonnances particuliers, pour l'entretien et la garde de leurs bois, vacants, bestiaux et généralement pour prendre soin de toutes leurs affaires, dans leur intérêt commun et dans celui de leurs paroisses, vics et dégairies": art. IV du titre I de la Coutume de Soule.
}

23 «Les paroissiens sont tenus de tenir et observer ces statuts et ordonnances pourvu qu'ils ne soient pas contraires au bien commun, et ne portent nul préjudice au Roi et à sess droits." : art. V.

24 Art. II. 
tulée: "Des libertés des régnicoles du présent royaume", est moins explicite et ne reconnaît que la liberté de réunion et de prendre des décisions obligatoires pour tous, "pourvu qu'elles ne soient pas au préjudice du Roi, de ses droits, ni contre le bien public" 25 .

Ces privilèges, auxquels il faut ajouter le droit de chasse et de pêche 26 d'avoir leurs propres moulins, fours et pressoirs, d'utiliser les eaux et de disposer de leurs terres allodiales..., y compris la décapitation pour dernier supplice 27 étaient communs à tous les Basques de part et d'autre de la frontière.

Chaque province jouissait d'une entière autonomie administrative, notamment financière, au sein du royaume de France comme d'Espagne. En Espagne, les Basques ne payaient d'impôts au Souverain que librement consentis par les Juntas ou les Cortes de Navarre. En France, le Labourd et la Soule étaient des pays abonnés et la Basse-Navarre un pays d'états.

Ayant leurs propres milices, composées de volontaires et commandées par des officiers nommés par les assemblées locales, les Basques étaient exemptés de servir dans les armées royales, bien qu'en France Louis XIV ait, en temps de guerre, procédé à de fréquentes réquisitions de marins et de charpentiers de navire.

En Espagne, les Basques disposaient d'une grande liberté testamentaire et matrimoniale ainsi que de la liberté commerciale ${ }^{28}$, la barrière douanière du royaume d'Espagne s'arrêtait à l'Ebre; les terres basques bénéficiè-rent de la franchise commerciale jusqu'en 1841. Ils pouvaient aussi exercer librement le métier de leur choix et accéder à toutes les charges y compris dans l'administration royale, dans l'armée ou autres fonctions interdites aux roturiers.

Les Basques jouissaient de ces privilèges, à une époque où la liberté, la plénitude de droits était le privilège de la noblesse. Si la noblesse est syno-

\footnotetext{
25 Art. II, rubrique 35 du For de Basse-Navarre.

26 Les restrictions qu'apporte la rubrique 31 du For de Basse-Navarre au droit de la chasse et de la pêche sont applicables à tous et n'ont pour objer que la protection du gibier et du poisson.
}

27 « Tout homme qui tue un autre doit être condamné à avoir la tête tranchée, s'il n'est qu'il l'eut fait en légitime défense": art. II, titre XIX de la Coutume de Labourd et art. II, titre 35 de la Coutume de Soule, alors qu'en France: "En crimes qui méritent la mort, le vilain sera pendu et le noble décapité": Antoine LOYSEL, Institutes coutumières, Slatkine reprints 1971, T. II, p. 218.

28 Chapitres XIV et XVI du Fueros viejo de Biscaye et loi X du titre I du Fueros nuevo. 
nyme non de chevalerie ou de vassalité, mais de liberté, alors l'affirmation de certains auteurs, tel Sanadon, évêque constitutionnel de Bayonne ${ }^{29}$, que tous les Basques étaient nobles, est exacte 30 .

C'est, d'ailleurs, ce critère de la liberté personnelle qui est invoqué par le roi de Castille quand il reconnut aux Basques la hidalguía universal (noblesse universelle). Il déclara "ce caractère d'hommes francs et ingénus, non sujets à la servitude, équivalent juridique de la noblesse". Il s'agissait d'une noblesse innée et non acquise, mais aussi d'une noblesse réelle, attachée à la terre ${ }^{31}$, et non personnelle. Les Juntas et la Diputación 32 de Biscaye s'opposèrent toujours à ce que le roi concédât des titres personnels de noblesse dans la Seigneurie ${ }^{33}$. Cependant, la noblesse des Biscayens, comme des Guipuzcoans, était inférieure à celle des hidalgos de Navarre, d'Alava ou de Castille.

Le principe selon lequel tout homme originaire de la province était noble fut reconnu par le roi de Castille aux habitants d'Alava en 1314, de Biscaye en 1394 et de Guipuzcoa en 148034 ainsi que par le roi de Navarre au XVe siècle, aux habitants des vallées navarraises. Dans les livres de feux de 1366 et 1427 , les habitants de la vallée du Baztán sont dits nobles, et dans un procès de 1437 à 1440, la vallée dont la noblesse était menacée se définit comme une communauté de "nobles, francs, ingénus et libres de toute servitude", ce qui fut reconnu par le roi de Navarre en 144035.

\footnotetext{
29 Don Barthélémy, Jean-Baptiste SANADON, Essai sur la noblesse des Basques, pour servir d'introducrion à l'histoire générale de ces peuples.s rédigé sur les Mémoires d'un militaire basque, par un ami de la nation, Pau 1785.

30 « seuls ils ont traversé ces long et douloureux siècles du moyen âge sans se laisser déshonorer par la servitude... et vraiment les Basques étaient tous nobles autant que les hauts barons des cours de France et d'Espagne, car leurs lois ne dépendaient pas d'un maître": Elisée RECLUS cité par Anne Marie LAGARDE, L'univers psychique des Basques, une instauration de la symérrie des sexes. Expression sociale el linguistique, thèse, UPPA, 2000, p. 94.
}

31 Cf. Maité LAFOURCADE, "Le statut juridique de la noblesse basque", in Autour de Bertrand d'Etchauz, évêque de Buyonne (fin XVe - début XVIII siècle), Actes du colloque des 12 et 18 septembre 1999, Bayonne 2000, p. 229-246.

32 Cf . au sujet de la diputación de Biscaye: Gregorio MONREAL CIA, Las instituciones..., op. cit, p. $423-427$.

33 Cf. Ignacio de URQUUJO, "La nobleza vizcaina a través de los Fueros", Zumárraga (3), Bilbao 1954, p. 77.

${ }^{34}$ Cf. Eugène GOYHENECHE, I R Pays Basque - Soule - Labourd - Basse-Navarre, SNERD, Pau 1979 , p. 248.

35 A. FLORISTAN et J. IMIZCOZ, "Sociedad y conflictos sociales (siglos XVI-XVIII", in Economia, sociedad y cultura durante el antiguo régimen, II Congreso mundial vasco, Congreso de historia de Euskal Herria, San Sebastián 1988, Tomo III, p. 281-308. 
Le Fueros de Biscaye déclare à plusieurs reprises la noblesse des Biscayens. Ainsi dans la loi XVI du titre I du Fuero Nuevo, on peut lire que "tous les naturels, voisins et habitants de cette dite Seigneurie de Biscaye, Tierra-Llana, Villas, Ciudad, Encartaciones y Durangueses ${ }^{36}$, sont de notoires nobles et jouissent de tous les privilèges des hommes nobles..." 37. Et quiconque venait habiter et s'établir en Biscaye devait fournir des preuves de limpieza de sangre (pureté de sang) ${ }^{38}$ afin qu'il puisse jouir de la qualité de noble.

Les agents du roi qui avaient le droit de haute justice, se plaignaient de ne pouvoir appliquer la torture aux Biscayens ${ }^{39}$, ce mode de preuve étant réservé aux roturiers.

Les Biscayens luttèrent pour conserver leur noblesse qui fut plusieurs fois menacée ${ }^{40}$ Les cahiers d'Hermandad et les Fueros de Guipuzcoa déclaraient de même la hidalguía des Guipuzcoans 41 et exigeaient de tout étranger qui voulait s'établir dans la province des preuves de pureté de sang.

La généralisation de la noblesse consacrait d'antiques libertés individuelles et était assortie d'importantes garanties judiciaires, tout-à-fait insolites au Moyen-âge, voire à l'époque moderne.

\footnotetext{
${ }^{36}$ Il s’agit des divers territoires qui composaient la Seigneurie; leurs fueros avaient été rédigés avant le Fueros general de Biscaye: Fueros Antiguo de la Merindad de Durango, XIve siècle; Fueros de Abellaneda, 1394; Fueros de las Encartaciones, 1503. Cf. Legislación foral de Bizkaia, op-cit. Le Fueros de Abellaneda de 1394 reconnaissait aux Biscayens le droit d'user de leur qualité de noble dans toutes les provinces espagnoles. De même, les cahiers d'Hermandad de Biscaye et des Encartaciones, de la dernière décade du XIve siècle, affirmaient la noblesse des habitants.
}

37 Legislación foral..., op-cit, p. 231.

38 Loi XIII du titre I, op-cir, p. 229.

39 Loi IX du titre IX, op-cit, p. 258.

40 Ainsi, au XVIe siècle, Juan García publia un livre intitulé: De Hispanorum nobilitate..., dans lequel il niait la noblesse biscayenne. Les Biscayens adressèrent une requête au roi et obtinrent de Philippe II une lettre, datée du 30 janvier i590, par laquelle il ordonnait la saisie de I'ouvrage afin que les textes contestés soient supprimés: Adrián CELAYA IBARRA, "Los fueros vascos y los derechos humanos", loc-cit, p. 14.

41 « ... la condition de noble empêchait dans le pays (en Guipuzcoa) l'application de la torture en aucun cas.. " : Cuaderno de Hermandad de Guipuzcoa de 1397, et chapitre II du titre II de la Nueva recopilación. 


\section{B. Les garanties judiciaires}

Tous les Fueros et Coutumiers du Pays basque et de Navarre s'opposaient à toute accusation et détention arbitraire, jusqu'au plus récent, celui de Basse-Navarre de 1611, dont l'article VI de la rubrique 18 déclare: "Nul ne sera contraint par corps, ni assigné par ajournement personnel sans information et décret préalable, si ce n'est en cas de flagrant délit et suspicion de fuite". Ce principe qui, aujourd'hui nous semble élémentaire ne fut reconnu en Europe occidentale qu'au XIX ${ }^{\text {e }}$ siècle. La loi anglaise de 1679 qui créa l'Habeas Corpus, ne donnait pas une formulation aussi catégorique que les Fueros basques, en particulier les Fueros de Biscaye ${ }^{42}$.

C'est en effet, surtout dans les Fueros de Biscaye 43 qu'est défini un étonnant système de garanties pénales qui, en de nombreux aspects, dépasse les constructions modernes du droit pénal, dit humanitaire, de nos jours. Il témoigne d'un profond respect de la personne humaine, mêmedélinquante 44 .

Les Biscayens ne pouvaient être jugés que par leurs "juges naturels" 45 Et s'ils étaient domiciliés hors de Biscaye, c'était le "juez mayor" de la Salle de Biscaye en la Chancellerie de Valladolid qui était le seul compétent ${ }^{46}$.

La procédure était accusatoire au civil et au pénal. Le juge était saisi sur plainte ou dénonciation. Mais il était interdit d'accuser nommément une personne. L'accusateur ne pouvait qu'indiquer le lieu, les jour et heure, et les circonstances du fait 47 avec les noms des témoins. C'est une garantie de la dignité humaine qui fait défaut dans nos législations modernes si soucieuses des droits de l'homme. Seul le fait était dénoncé et c'était le juge qui devait déterminer l'auteur ${ }^{48}$.

\footnotetext{
42 Chapitre LXIII du Fueros Viejo et loi XXVI du titre 11 du Fueros Nuevo.

$43 \mathrm{Cf}$. sur le droit pénal biscayen: Jesús de GALINDEZ, La legislacón penal en Viscaya, Bilbao 1934 ; A. PEREZ AGOTE, "El contenido penal del Fueros Viejo de Viscaya de 1452", Estudios vizcaínos, 1972, III, p. 379-390; M.V. CABIECES IBARRONDO, "La pena de muerte en el señorío de Viscaya", Estudios de Deusto, 1979, XXVII, p. 221.303

44 Chapitres LXI à LXVIII du Fueros Viejo et titres 8 à 11 du Fueros Nuevo.

45 Chapitres XIII et XX du Fuero Viejo, loi XIX du titre I et titre II du Fuero Nuevo.

${ }^{46}$ Loi XX du titre I du Fuero Nuevo. La Sala de Viscaya de la Real Chancillería de Valladolid fut créée en 1385 .

${ }^{47}$ Loi I du titre IX du Fuero Nuevo.

48 Chapitres XXVI, XXXVI, L du Fuero Viejo; lois I et s. du titre IX du Fuero Nuevo.
} 
Après enquête, le juge prononçait une sentence d'appel sous l'arbre de Guernica ${ }^{49}$. Il affichait une convocation de l'accusé à Guernica et une autre dans la paroisse de sa résidence, par laquelle il le sommait de se présenter dans un délai de trente jours à la prison de son choix ${ }^{50}$. Pendant ce délai, l'accusé était libre et le juge ne pouvait pas l'incarcérer. La prison n'était prononcée que si le prévenu ne se présentait pas ${ }^{51}$.

En 1506, le corregidor 52 ayant réclamé la réforme de cette disposition du Fueros, les juntas la refusèrent; tout au plus, le Fueros réformé de 1526 prévoyait des exceptions pour les cas de flagrant délit et autres cas, assez nombreux il est vrai, précisés dans la loi I du titre 853 .

L'accusé qui répondait à la convocation du juge avait le droit de recevoir copie de toutes les pièces du procès, des charges qui pesaient sur lui comme des déclarations et dépositions des témoins avec leurs noms ${ }^{54}$, ce qui était tout-à-fait exceptionnel à une époque où dominait la procédure inquisitoire et secrète.

Il pouvait fournir des preuves à sa décharge par tous moyens 55 , prouvant notamment la fausseté des témoignages. Mais l'accusateur, lui, ne pouvait plus fournir aucune preuve; dès lors que l'accusé s'était présenté, l'accusation était close ${ }^{56}$. Ne restait que la défense, alors que la procédure inquisitoire partout en usage à cette époque recourait à tous les moyens, dont la torture, pour obtenir des aveux 57.

49 Chapitres XXXXİ et LII du Fuero Viejo, loi Vdu titre IX du Fuero Nuevo.

50 Loi VI du titre IX et lois I et II du titre 11 du Fuero Nuevo. L'accusé pouvait choisir entre les deux prisons de Biscaye, l'une étant à Guemica et l'autre au lieu de résidence du corregidor ou de son lieutenant.

51 Chapitre LII du Fuero Viejo et Ioi V du titre Ix du Fuero Nuevo.

52 Le corregidor était l'agent du roi dans les provinces. Créés par Alphonse XI, puis généralisés, comme en France, dans la seconde moitié du XVIe siècle, les corregidores furent supprimés en 1835.

53 « ...vols, viols, meurtre d'un étranger sans aucun parent dans la Seigneurie, mendiants, femmes notoirement dévergondées, ceux qui troublent le voisinage et disent des couplets et chansons à la manière de libelle infamant, entremetteurs, sorciers et sorcières, hérétiques, en cas de crime de lèse majesté, fabricants de fausse monnaie et auteurs de crimes contre nature". La loi V du Titre 9 ajoute des précisions.

54. Chapitre L du Fuero Viejo et loi VII du titre 11 du Fuero Nuevo.

55 Lois XIX du titre 11 du Fueros Nuevo.

56 Loi IX du Titre 11 du Fueros Nuevo.

57 Cf. notamment Yvonne BONGERT, "Le pro/modo probationum", Revue Historique de Droit français et étranger, 78 (1), janvier-mars 2000, p. 16; "Le juge et la question dans la doctrine du XYIc siècle", Mélanges Ankum, p. 37-58; "Réflexions sur l'administration de la justice criminelle en France au XVIIII siècle", Mélanges Sturm, I, 1999, p. 617-639. 
En Pays basque, la torture était prohibée. "Pour aucun délit ou méfait, public ou privé, grand ou léger, de quelque importance ou gravité qu'il soit..., qu'on ne soumette à la question ou qu'on ne menace de torture, directe ou indirecte, aucun Biscayen, en Biscaye ou à l'extérieur, en aucun lieu", peut-on lire dans la loi XII du titre I du Fueros Nuevo ${ }^{58}$ Et cette disposition était respectée; en cas de plainte adressée au roi, celui-ci intervenait immédiatement auprès du corregidor pour renouveler l'interdiction et punir le coupable 59 .

Le Fueros de Guipuzcoa qui, dans le chapitre x du titre 13 interdisait aussi la torture, se référait à la noblesse des Guipuzcoans.

De plus, ceux qui étaient accusés pour une cause, ne pouvaient être poursuivis pour une autre affaire jusqu'à ce que leur procès soit jugé et qu'ils soient remis en liberté 60 .

Ajoutons que les prisonniers qui fournissaient une caution idoine devaient être libérés 61 .

L'appel était toujours possible, il se faisait devant le corregidor 62 et dans les causes criminelles devant le juez mayor de la chancellerie de Valladolid; il était suspensif d'exécution 63. En cas de condamnation à "mort, effusion de sang, mutilation de membres, condamnation à une peine pécuniaire de plus de trois mille maravedis", de la sentence du lieutenant général du corregidor, on pouvait faire appel devant le corregidor et les députés de Biscaye conjointement 64 .

Lorsque la condamnation à mort ou autre peine assortie de la confiscation des biens du condamné était prononcée, ses biens de famille dits "raíces", situés en Biscaye, ne pouvaient être saisis, parce que la propriété en

\footnotetext{
58 Cette prohibition est répétée dans la loi IX du titre IX qui prévoit une exception "pour les crimes d'hérésie, lèse-majesté, falsification de monnaie et péché contre nature qui est la sodomie".

59 "Provision de la reine Doña Juana au corregidor de Biscaye lui interdisant d'infliger la question à ceux qui étaient mis en accusation, 1514", in Estanislao Jaime de LABAYRU, Historia general del Señorio de Viscaya, Bilbao, Tome IV, 1968, p. 61.

60 Chapitre LIII du Fueros Viejo et loi V du titre 11 du Fueros Nuevo.

${ }^{61}$ Loi III du titre rx et loi XX du titre 11 du Fueros Nuevo.

62 Loi II du titre XIX.

63 Loi I du titre 3.
}

64 Loi XI du titre 29. Cf. au sujet de la peine de mort en Biscaye: Maria Vitoria CABIECES IBARRONDO, "La pena de muerte en el Señorío de Viscaya", loc-cit, p. 265-271. 
Pays basque était collective et que la maison ancestrale avec ses appartenances et dépendances, meubles et immeubles, appartenait à la famille et non à un seul individu qui n'en était que le gestionnaire avec son conjoint et ses père et mère pendant une génération.

Enfin, les Biscayens ${ }^{65}$, Alavais ${ }^{66}$, Guipuzcoans 67 ne pouvaient pas être arrêtés pour dette ordinaire. Leur maison, leurs armes, leurs chevaux ne pouvaient être saisis. Si cette règle n'était pas respectée, le roi intervenait pour la rappeler aux juges 68 . En conséquence, aucun exécuteur de justice ne pouvait s'approcher à moins de huit brasses de la maison d'un débiteur sans l'accord de son maître, à moins qu'il ne fût sans armes et accompagné d'un greffier pour l'unique objet d'inventorier les biens séquestrables 69 .

La maison d'un Basque était inviolable. L'exception admise était lorsque l'exécuteur montrait le mandement judiciaire pour prendre un malfaiteur qui s'y était réfugié.

Ces garanties judiciaires, étaient communes à tous les Basques. Le Pays basque avait devancé de plusieurs siècles l'égalité juridique préconisée par la Révolution française.

\section{L'égalité juridique}

A une époque où la liberté, la plénitude des droits étaient l'apanage de la noblesse, cette égalité est tout-à-fait remarquable. Mais alors que les Fueros privilégiaient la liberté, les Coutumiers du Pays basque français accordaient plus d'importance à l'égalité. Elle se retrouve tant en droit public qu'en droit privé.

65 Loi III du titre 16 du Fueros Nuevo.

66 Chapitre XXXVIII du Fueros de Ayala de 1373. En 1489, la terre d'Ayala accepta le droit castillan; mais ses habitants firent trois réserves concemant des règles traditionnelles auxquelles ils ne voulaient pas renoncer. La première était que "le voisin d'Ayala ne soit pas mis en prison pour dette".

67 Art. IV-2 du Fueros, Cf. José Luís BANUS y AGUIRRE, El Fuero de San Sebastián, Ediciones del Excmo. Ayuntamiento de San Sebastian, 1963, p. 61

68 « Provision du Roi Catholique pour qu'on n'emprisonne aucun Biscayen pour dette civile 1475", Estanislao Jaime de LABAYRU, Historia general..., op-cit, Tome III, 1968, p. 662-663.

69 Chapitre LXXVII du Fueros Viejo; la loi IV du Titre 16 du Fueros Nuevo interdit aux exécuteurs de justice de s'approcher de la maison à moins de quatre brasses, contre la volonté de son maître. 


\section{A. La démocratie basque}

Les Basques, à l'exception des Navarrais, n'ont pas connu la division de la société en ordres. En Espagne, ils étaient tous nobles et, en. France, nobles et non nobles avaient le même statut juridique; seules les règles successorales les distinguaient, le privilège de masculinité ayant, à l'époque de la rédaction des coutumes, pénétré en Pays basque ${ }^{70}$.

Une enquête réalisée en Labourd par le roi Edouard II d'Angleterre duc d'Aquitaine, en $1311^{71}$, révèle que tous les habitants, nobles et non nobles, sont placés sur un pied d'égalité vis-à-vis du seigneur-roi. Tous lui devaient également l'hommage et les services vassaliques, dont les services d'ost et de chevauchée, précisés dans l'enquête. Seule, l'albergade due par les hommes liges du roi à ce demier et à son bailli distinguait les nobles des autres habitants 72 .

Cette égalité subsista après l'annexion du Labourd par Charles VII. Et, si une inégalité s'est installée, c'est au détriment de la noblesse. Sous l'Ancien Régime, non seulement elle ne jouissait que de privilèges honorifiques, mais encore elle était frappée d'incapacités. Les nobles labourdins n'avaient aucun droit sur les terres communes, ne pouvaient exercer aucune fonction municipale et étaient exclus tant des assemblées paroissiales que du Biltzar qui était l'assemblée générale de la province ${ }^{73}$.

Dans l'article 38 de leur cahier de doléances rédigé pour les Etatsgénéraux de 1789, les nobles se plaignaient de n'avoir "aucune part à l'administration du Pays" et de payer "la huitième partie des impositions", alors

\footnotetext{
70 Articles I et II du titre 12 de la Coutume de Labourd. En Soule et en Basse-Navare, il avait même pénétré dans le régime successoral de certaines maisons franches: la rubrique 27 de la Coutume de Soule énumère les maisons, précisant leur régime successoral: droit d'aînesse sans distinction de sexe ou avec privilège de masculinité. En Basse-Navarre, le droit d'aînesse absolu ne prévalait que pour les maisons fivatières et les maisons franches des vallées navarraises; un règlement de 1705 décida que l'état des maisons serait rédigé mais il ne l'a jamais été; ce qui fut la source de nombreux procès.

71 A.M. Bayonne: DD 20-1. Cf. Maité LAFOURCADE, "La féodalité en Labourd. Enquête ordonnée par Edouard II d'Angleterre pour connaître ses droits sur cette terre -131I", Eugène Goyheneche. Hommage, Fusko-Ikaskuntza, Saint Sébastien 2001, p.165-179.

72 Ce service était racheté par les hommes-liges du roi deux sous morlans par an.

73 Maité LAFOURCADE, "Le statut juridique...", loc-cit.
} 
que "les biens nobles se forment pas la vingtième partie des fonds $74 \ldots$ ». Et, en 1789, c'étaient les nobles qui réclamaient l'égalité des droits! 75

Le Biltzar ${ }^{76}$ représentait le type même des assemblées démocratiques basques. La noblesse et le clergé en étaient exclus. Il était composé des seuls députés des trente-cinq paroisses du pays, lesquels, élus par les maîtres de maison de leur paroisse, étaient munis d'un mandat impératif. Le Biltzar comprenait deux sessions afin que les députés puissent, après l'exposé des questions par le syndic lors d'une première session, revenir dans leur paroisse respective recueillir le vote des maîtres de maison, chaque maison, quelle que fût son importance, ayant une voix. Il s'agissait d'une démocratie directe à base familiale.

En Biscaye et Guipuzcoa, le territoire étant plus vaste, le suffrage était indirect à deux ou trois degrés et le mandat des députés aux Juntas était représentatif; mais, à la base, tous les maîtres de maison votaient également 77 . Il n'y avait aucune distinction entre nobles et roturiers, et le clergé était exclu 78 non seulement parce que les clercs n'étaient pas maîtres de maison, mais aussi par crainte de l'influence de cet ordre tout puissant.

En Soule, la noblesse et le clergé étaient parvenus, à une époque inconnue, à s'ajouter au Silviet qui, analogue au Biltzar, était l'assemblée démocratique basque. Le Grand Corps, noblesse et clergé réunis, avait une voix et le Silviet une voix, de telle sorte que la Soule avait une organisation intermédiaire entre celle, traditionnelle du Labourd, de Biscaye et du Guipuzcoa, et celle, de droit commun, de la Navarre et de la Basse-Navarre. Aux Cortes de Navarre, comme aux Etats de Basse-Navarre, créés en 1523, les trois ordres étaient représentés, chacun ayant une voix ${ }^{79}$. Toutefois, dans

\footnotetext{
74 Pierre YTURBIDE, Cahiers des doléances de Bayonne et du Pays de Labourd pour les Etats Généraux de 1789, Bayonne, 1912, p. 18.

75 Circulaire (imprimée) rédigée par les membres de la noblesse du bailliage de Labourd demañdant l'abolition des privilèges et invitant le peuple à l'union: AD-PA C 1613.

76 Cf. au sujet du Biltzar: Maite LAFOURCADE, "Le Bilçar du Pays de Laboürd au XVIII" siècle", De la res publica a los Estados modernos, Actes des joumées internationales d'Histoire du droit de Saint-Sébastien 31 mai-3 juin 1990, Universidad del País Vasco, San Sebastián, 1992, p. 125-147.

77 Cf. Gregorio MONREAL CIA, Las instituciones públicas..., op. cit, p. 141-326

78 L'entrée des clercs aux Juntas était expressément interdite par le Fueros de Guipuzcoa: chapitre iv du titre 18.
}

79 Cf. Maïté LAFOURCADE, "Les assemblées provinciales du Pays Basque français sous l'Ancien Régime", Lapurdum IV, Rev. de l'UMR 5478 du CNRS, Bayonne 1999, p. 303-329. 
les vallées navarraises, subsistait l'antique régime démocratique basque dans lequel les maisons étaient égales et le clergé exclu.

Ajoutons qu'il n'y avait aucune discrimination sociale, aucun mépris des nobles à l'égard des laboureurs et aucune haine de ces derniers à l'égard des nobles. Comme le déclarait Dominique Joseph Garat, député du tiers état labourdin aux Etats généraux de 1789, dans un mémoire lu en 1784 à l'académie de Bordeaux: 'Le laboureur, fier de ce titre qu' on lui donne et qu'il prend dans toutes les occasions comme un titre d'honneur, s'assied à la table du gentilhomme et s'y croit à sa place, et le gentilhomme pense comme lui" 80 .

Cette égalité existait aussi au sein de chaque maison, en droit familial.

\section{L'égalité en droit familial}

Dès les premiers documents écrits concernant les Vascons, dans les Fueros et les Coutumiers comme dans les actes de la pratique, la femme basque apparaît comme l'égale de l'homme. D'ailleurs, la langue basque, l'euskara, ne reconnaît pas de distinction entre le masculin et le féminin.

Le droit successoral ne faisait aucune différence entre les filles et les garçons. Dans les provinces basques d'Espagne, les parents choisissaient indifféremment parmi leurs enfants, celui ou celle qui était le plus apte à bien gérer le patrimoine familial pour en faire leur héritier 81 . Les biens "raíces" appartenaient à la famille; ils devaient se perpétuer comme elle; ils étaient donc inaliénables et indivisibles. Ils ne devaient avoir qu'un seul héritier à chaque génération. Le terme "héritier" est, d'ailleurs, inexact; les Basques l'appelaient etxerakoa: "celui qui est pour la maison", en espagnol: "el destinado a casa". Il était le gérant du patrimoine familial pendant une génération et le responsable de tous les membres de la famille, vivants et morts; il devait notamment donner à ses frères et sœurs une situation digne de la maison et loger, nourrir et entretenir tous ceux qui ne l'avaient pas quittée. Ii en

80 Mémoire sur Bayonne el les Basques, manuscrit du XIX̄e siècle: BM Bayonne, ancien fonds $94-$ 26.

81 Dans les provinces basques espagnoles, la liberté de tester était très grande: titre 21 du Fueras Nuevo de Biscaye. Les parents désignaient et désignent toujours de nos jours, l'héritier ou héritière des biens "raíces" par un testament en main commune ou par commissaire; cette forme de testament, prohibé tant en droit espagnol que français, était très répandue. Cf. au sujet de la liberté de tester en Biscaye: Itziar MONASTERIO ASPIRI, Los pactos sucesorios en el derecho vizcaíno, Bilbao 1994. Dans le Fuero de Ayala, la liberté de tester était absolue: chapitre XXVIII. 
était de même dans les provinces basques françaises.

Dans ces dernières, le droit d'aînesse, sans distinction de sexe, s'était imposé. L'aîné, fille ou garçon ${ }^{82}$, héritait des biens dits papoaux ou avitins, c'est-à-dire qui étaient dans la famille depuis au moins deux générations, meubles et immeubles étant confondus. $\mathrm{Si}$, en biens nobles le privilège de masculinité avait pénétré en Pays basque français, après cette concession faite au droit nobiliaire, le principe basque de l'égalité des sexes reprenait ses droits: en cas de mariages successifs, si du premier il n'y avait que des filles, l'aînée de celles-ci excluait les enfants des mariages subséquents, même s'il y avait des mâles 83 .

Un héritier épousait généralement une cadette d'une autre maison et une héritière un cadet ${ }^{84}$. Dans le couple alors que partout en France, sous l'Ancien Régime, régnait le principe de l'incapacité juridique de la femme mariée, en Pays basque mari et femme avaient des droits égaux. Ils géraient conjointement le patrimoine familial. Tout acte de disposition d'un bien de famille nécessitait leur commun consentement ${ }^{85}$; le Fueros de Biscaye appelait cette association entre mari et femme, Hermandad y companía 86.

La voix de la femme, si elle était l'héritière, l'emportait même sur celle de son mari, en cas de désaccord entre eux, notamment pour consentir au mariage de leur enfant aîné 87.

Cependant le mari était, comme en droit commun coutumier, le chef de la communauté d'acquêts. Mais, après cette atteinte au principe basque de l'égalité des sexes, les Coutumes de Labourd et de Soüle précisaient que le

82 Art. III du titre 12 de la Coutume de Labourd, Cf. Maïté LAFOURCADE, "Droit successoral et droit matrimonial en. Pays basque sous l'Ancien Régime", Le droit de la famille et l'Europe, Actes des journées internationales d'Histoire du droit, Strasbourg, 23 au 26 mai 1991, P.U. Strasbourg 1992, p. 517-529.

83 Art. II du titre 12 de la Coutume de Labourd, art. II du Titre 27 de la Coutume de Soule et art III de la rubrique 27 du For de Basse-Navarre.

84 En Labourd, de 1774 à 1789 , il y eut $82,42 \%$ de contrats de mariage entre un enfant héritier et un enfant cadet, dont $51 \%$ d'héritiers et $49 \%$ d'héritières. Les mariages entre deux enfants héritiers qui avaient pour conséquence la fusion de deux domaines, étaient très rares: 2,91 \%: Maïté LAFOURCADE, Mariages en Labourd sous l'Ancien Régime, Univ. du Pays basque, Leioa 1989, p. 38-39.

85 Art. VI du titre $\mathrm{xx}$ de la Coutume de Labourd: "Le mary ne peult faire aucune vente ne aliénation des biens assignés au mariage, si la femme ne y consent, ne aussi la femme sans le consentement du mary". De même, article V du Titre 24 de la Coutume de Soule.

86 Loi IX du titre 20 du Fueros Nuevo de Biscaye.

87 Art. X du titre 12 de la Coutume de Labourd. 
mari ne pouvait disposer sans le consentement de sa femme des biens qu'elle avait acquis par son travail ${ }^{88}$. La femme pouvait même en disposer seule, sans l'autorisation de son mari, quand elle était marchande et pour les besoins de son commerce, pour l'entretien des biens assignés en mariage, c'est-à-dire du patrimoine familial de celui des deux qui était l'héritier et de la dot apportée par son conjoint, et pour la nourriture des enfants nés du mariage ${ }^{89}$. Et, en dehors de ces trois hypothèses, l'obligation contractée par la femme seule n'était pas nulle, elle était seulement suspendue jusqu'à la mort du mari 90.

La femme basque était encore au XvIII ${ }^{\mathrm{e}}$ siècle, comme au Moyen Âge, la socia mariti. Le principe de l'incapacité juridique de la femme mariée n'avait qu'imparfaitement pénétré en Pays Basque où ill se heurtait au principe basque de l'égalité des sexes.

Cette égalité existait aussi entre les deux couples de gestionnaires du patrimoine familial, voire les trois si les grands-parents survivaient, soit l'héritier ou héritière de chaque génération et son conjoint qui avait porté une dot dans la maison dans laquelle il entrait par mariage. Les deux couples étaient appelés dans les actes de la pratique, "maîtres vieux" et "maîtres jeunes". C'est le régime typiquement basque de la "coseigneurie", que les Basques d'Espagne appellent la comunicación foral ${ }^{91}$. Tous avaient des droits égaux, quel que fût son sexe ou sa qualité, héritier ou dotal. Les actes d'administration, et à plus forte raison, les actes de disposition, nécessitaient le consentement de tous les indivisaires 92 . L'égalité entre les deux couples était telle qu'en cas de mésentente, chaque couple pouvait demander le partage du patrimoine familial, lequel se faisait par moitiés égales; mais chaque couple n'avait que la jouissance et administration des biens de son lot, sans pouvoir

\footnotetext{
88 Art. II du titre Ix de la Coutume de Labourd et art. II de la rubrique 24 de la Coutume de Soule. Le For de Basse-Navarre ne prévoyait pas cette liberté de Ia femme exerçant une profession : art. II de la rubrique 25 .

89 Art. IX du titre IX de la Coutume de Labourd et article VIII de la rubrique 24 de la Coutume de Soule. Le For de Basse-Navarre ne prévoit pas cette éventualité.

90 Art. X du titre xX de la Coutume de Labourd et art. IX de la rubrique 24 de la Coutume de Soule. 91 Chapitre XCVI du Fuero Viejo de Biscaye et loi I du titre 20 du Fueros Nuevo.

92 Art. I et VI du titre 5 de la Coutume de Labourd et articles I et IV du titre 17 de la Coutume de Soule.
} 
en disposer sans le consentement de l'autre couple ${ }^{93}$.

A une époque où l'autorité du chef de famille était toute puissante, cette institution de la coseigneurie témoigne de l'esprit égalitaire des coutumes basques.

Mais la tendance unificatrice et centralisatrice de la Monarchie, beaucoup plus précoce en France qu'en Espagne ${ }^{94}$, entraîna la décadence des particularismes locaux.

La Révolution de 1789 en France acheva l'œuvre unificatrice de la Monarchie. Paradoxalement, les Basques, de tous temps libres, égaux et naturellement démocrates, perdirent, au nom de ces mêmes idéaux proclamés dans la Déclaration des droits de l'homme du 26 août, leurs libertés, virent s'installer au sein de leur communauté une oligarchie bourgeoise, source d'inégalités, et, la plupart trop pauvres pour pouvoir voter dans un régime censitaire, ne prirent même pas part aux élections.

La loi, "expression de la volonté générale", se substitua au droit coutumier et le Code civil de Napoléon imposa à tous les Français un droit unique, individualiste et bourgeois, aux antipodes du régime juridique basque.

En Espagne, les Fueros furent supprimés en Navarre en 1841 et, dans les autres provinces, en 1876, après l'échec des guerres carlistes. Mais, après la mort de Franco, la Constitution de 1978, tout en proclamant l'unité de la Nation espagnole, reconnut la pluralité des nationalités et des régions, leur reconnaissant un statut d'autonomie, avec pouvoir de légiférer. Dès lors, le régime foral fut rétabli, notamment en Pays basque et en Navarre.

Désormais, les Basques ont, en Espagne, leur propre droit. La dernière actualisation du Fueros de Navarre date du 1er avril 1987 et la loi du 1er juillet 1992, la ley del derecho civil foral del País Vasco, dans la communauté autonome basque, a adapté les vieux Fueros de Biscaye et d'Ayala à la société actuelle 95 . Une annexe concernant le Guipuzcoa dont les coutumes

93 Art. XVIII et s. du titre ix de la Coutume de Labourd. En Soule, le survivant adventice ou dotal de chaque couple n' avait droit qu'au quart du patrimoine familial : art. XXII et s. du titre 24 de Ia Coutume. En Basse-Navarre, chaque couple pouvait disposer de sa part: art. XI de la rubrique 24 du For; mais "les rédacteurs du For n'ont pas été unanimes sur les dispositions relatives à la coseigneurie", certains voulant restreindre l'article à la jouissance seulement de la moitié des biens: Jean GOYENETCHE, For et coutumes..., op-cit, p. 332, note 169.

94 Ce n'est qu'avec l'arrivée des Bourbons sur le trône d'Espagne, en 1700 que la tendance centralisatrice et unificatrice de la Monarchie en Espagne fut vraiment effective. 
relatives au droit privé n'avaient pas été rédigées, a été ajoutée à cette loi, le 16 novembre 1999.

Ces lois maintiennent les principes fondamentaux et l'esprit du droit basque, notamment la troncalidad, soit l'intégrité du patrimoine familial, inaliénable et indivisible, et la liberté testamentaire, du moins en partie. En France, le droit basque survit toujours, mais à l'état d'usages 96 . Toutefois, lois forales et usages ne concernent désormais que le droit de la famille, le droit pénal étant depuis longtemps en Espagne comme en France du domaine du droit public et de l'État qui se veut le seul grand protecteur des droits de l'homme.

Mais les Droits de l'homme sont-ils universels, comme on le prétend? Chaque société a droit à la reconnaissance de son identité, à la différence. "Toutes les cultures aspirent à des préoccupations et des valeurs fondamentales, mais l'universalisme culturel, en tant que position philosophique, est une erreur" 97. Il faut prendre garde aux dangers de l'impérialisme culturel qui fait "partie de la trajectoire historique de la modernité occidentale" 98.

\section{RESUME}

Deux cents ans avant la Pétition of Rights de 1648 et la loi anglaise de 1679 , bien avant les déclarations française et américaine des Droits de l'homme, les Fors basques, particulièrement les Fors de Biscaye de 1452 et 1526 , élaborés par les Juntas, soit le peuple lui-même, en dehors de toute orientation systématique, renferment des dispositions, issues d'usages ancestraux, garantissant les droits naturels de l'homme: la liberté et l'égalité. Généralement ignorés en dehors du Pays basque, ils méritent cependant une place, toute modeste qu'elle soit, dans la liste universelle des Droits de l'homme.

Les lois forales, datant du Moyen Age, répondent aux mêmes exigences humaines, aux mêmes inquiétudes que les déclarations des derniers siècles. De plus, elles proclament des libertés concrètes suivies d'effets, et non des libertés abstraites qui, en France, ont abouti à Robespierre et Napoléon...

\footnotetext{
95 Cette loi a été publiée, traduite en français, dans : La pratique actuelle du droit coutumier en Pays Basque, Actes du colloque tenu à Bayonne le 3 septembre 1994, P.U. Bordeaux 1998, p. 131-163. 96 Op-précit.

97 Boaventura de SOUSA SANTOS, "MulticultureIle des droits de l"homme", Droit et Société (35), 1997, p. 88

98 Article précit., p. 95.
} 